University of Nebraska - Lincoln

DigitalCommons@University of Nebraska - Lincoln

$9-2010$

\title{
Magnetoelectric effects in ferromagnetic cobalt/ferroelectric copolymer multilayer films
}

\author{
Abhijit Mardana \\ University of Nebraska-Lincoln \\ Mengjun Bai \\ University of Missouri-Columbia, baime@missouri.edu
}

A. Baruth

University of Nebraska-Lincoln

Stephen Ducharme

University of Nebraska, sducharme1@unl.edu

Shireen Adenwalla

University of Nebraska - Lincoln, sadenwalla1@unl.edu

Follow this and additional works at: https://digitalcommons.unl.edu/physicsducharme

Part of the Physics Commons

Mardana, Abhijit; Bai, Mengjun; Baruth, A.; Ducharme, Stephen; and Adenwalla, Shireen, "Magnetoelectric effects in ferromagnetic cobalt/ferroelectric copolymer multilayer films" (2010). Stephen Ducharme Publications. 69.

https://digitalcommons.unl.edu/physicsducharme/69

This Article is brought to you for free and open access by the Research Papers in Physics and Astronomy at DigitalCommons@University of Nebraska - Lincoln. It has been accepted for inclusion in Stephen Ducharme Publications by an authorized administrator of DigitalCommons@University of Nebraska - Lincoln. 


\title{
Magnetoelectric effects in ferromagnetic cobalt/ferroelectric copolymer multilayer films
}

\author{
A. Mardana, Mengjun Bai, ${ }^{a)}$ A. Baruth, Stephen Ducharme, and S. Adenwallab) \\ Department of Physics and Astronomy, The Nebraska Center for Materials and Nanoscience, \\ University of Nebraska-Lincoln, Lincoln, Nebraska 68588-0299, USA
}

(Received 4 June 2010; accepted 21 August 2010; published online 14 September 2010)

\begin{abstract}
Interactions between a ferromagnet, cobalt, and a ferroelectric copolymer, poly(vinylidene fluoride with trifluoroethylene) in thin film heterostructures result in a 5\% change in the ferroelectric polarization on application of a perpendicular $6 \mathrm{kG}$ magnetic field corresponding to a magnetoelectric coupling coefficient of $\alpha=5.45 \mathrm{~V} / \mathrm{cm}$ Oe. The effect disappears on magnetic saturation, ruling out conventional strain coupling. A simple model posits that the ferroelectric film develops in-plane strain gradients, a consequence of the coupling to strain gradients present at the domain walls in the multidomain Co layer, resulting in the measured polarization change via the flexoelectric effect. (C) 2010 American Institute of Physics. [doi:10.1063/1.3488814]
\end{abstract}

The magnetoelectric (ME) effect and its converse refer to the control of electric polarization and magnetization by magnetic or electric fields, respectively. The wide range of potential applications ${ }^{1}$ ranging from memory devices to magnetic field sensors are driving the exploration of multiferroic materials with larger ME coefficients than have previously been seen. ${ }^{2}$

Heterostructured materials with separate magnetic (or magnetostrictive) and ferroelectric components relax the competing demands and constraints on a single material. ${ }^{3-6}$ Efforts have focused on magnetic materials with the largest possible magnetostriction in order to maximize the piezoelectric/magnetostrictive coupling. Magnetic flux concentration has resulted in ME coefficients as high as 21.46 $\mathrm{V} / \mathrm{cm} \mathrm{Oe}^{7}{ }^{7}$ Thermally mediated effects ${ }^{8}$ in a relaxor ferroelectric polymer result in ME coefficients of $0.9 \mathrm{~V} / \mathrm{cm}$ Oe.

This manuscript reports results that suggest a different mechanism for ME coupling in a ferromagnetic/ferroelectric heterostructure, viz. the strain gradient created near magnetic domain boundaries in a multidomain magnetic film and its effect on the ferroelectric polarization through the flexoelectric effect. An organic ferroelectric, the copolymers of $70 \%$ vinylidiene fluoride (VDF) with $30 \%$ trifluroethylene (TrFE) i.e., $[\mathrm{P}(\mathrm{VDF}-\mathrm{TrFE} 70: 30]$ is overlaid with a transition-metal ferromagnet, Cobalt $(\mathrm{Co})$ in a heterostructure with thicknesses that are controllable at the atomic level with films in intimate contact.

The sample [Fig. 1(a)] consists of [glass/Al $(32.4 \mathrm{~nm}) /$ $\mathrm{P}(\mathrm{VDF}-\mathrm{TrFE})(45 \mathrm{~nm}) / \mathrm{Al}(2.9 \mathrm{~nm}) / \mathrm{Co}(10 \mathrm{~nm}) / \mathrm{Al}(25.6$ $\mathrm{nm})$ ]. The metallic layers were made from either evaporated $\mathrm{Al}$ or sputtered Co deposited through shadow masks $1 \mathrm{~mm}$ wide and $15 \mathrm{~mm}$ long, with the upper and lower electrodes (see inset b of Fig. 1), perpendicular to each other defining four spots with an overlapping electrode area of $1 \mathrm{~mm}^{2}$. Both Al electrodes were deposited at a rate of $1.2 \AA$ s. The top Al electrode served to prevent the diffusion of Co atoms into the soft polymer film. The upper Co layer was sputtered at low power, at a deposition rate of $0.056 \AA / \mathrm{s}$, with frequent pauses, to prevent shorting through the soft polymer layer.

\footnotetext{
${ }^{a)}$ Presently at University of Missouri-Columbia.

${ }^{b)}$ Electronic mail: sadenwalla1@unlnotes.unl.edu.
}

The [P(VDF-TrFE 70:30] layer was deposited by the Langmuir-Blodgett (LB) method, which results in films with superior crystallinity, excellent ferroelectric properties, ${ }^{9}$ and a saturation polarization of up to $0.1 \mathrm{C} / \mathrm{m}^{2} .{ }^{10}$ Prior to deposition of the top electrode, the ferroelectric polymer film is annealed at $130{ }^{\circ} \mathrm{C}$ for an hour in air, to increase its crystallinity. Copper wires were attached to both electrodes with silver paint, allowing for pyroelectric measurements and enabling polarization switching. X-ray diffraction measurements indicate that the polymer grows in the (111) direction resulting in a polarization vector at $30^{\circ}$ to the surface normal. ${ }^{11}$ The in-plane structure consists of small crystallites, $30-50 \mathrm{~nm}$ in size ${ }^{12}$ with the polarization vectors for the various crystallites forming a cone at $30^{\circ}$ around the normal, resulting in a net macroscopic polarization along the normal.

Ferroelectric polarization is measured using the Chynoweth modulation method, ${ }^{13}$ for pyroelectric current, which is proportional to the normal component of the film polarization, irrespective of polarization state. Moreover it is perturbative and does not alter the polarization state. ${ }^{14}$ In this method, the sample temperature was modulated by a $3 \mathrm{~mW}$ helium-neon laser operating at a wavelength of $633 \mathrm{~nm}$ and an optical chopper at $2 \mathrm{kHz}$. The resulting pyroelectric cur-

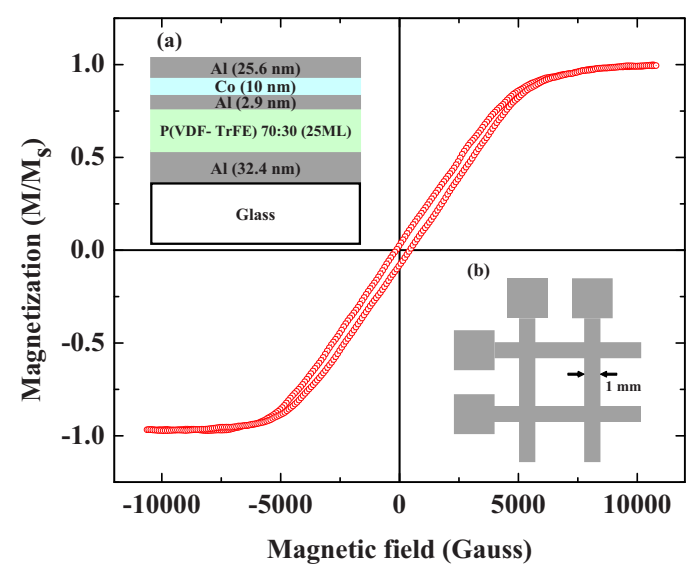

FIG. 1. (Color online) The out-of-plane hard axis magnetic hysteresis loop of the $10 \mathrm{~nm}$ Co electrode measured by PMOKE. Upper inset: Schematic of the heterostructure. Lower inset: top view of the sample showing the electrode geometry. 


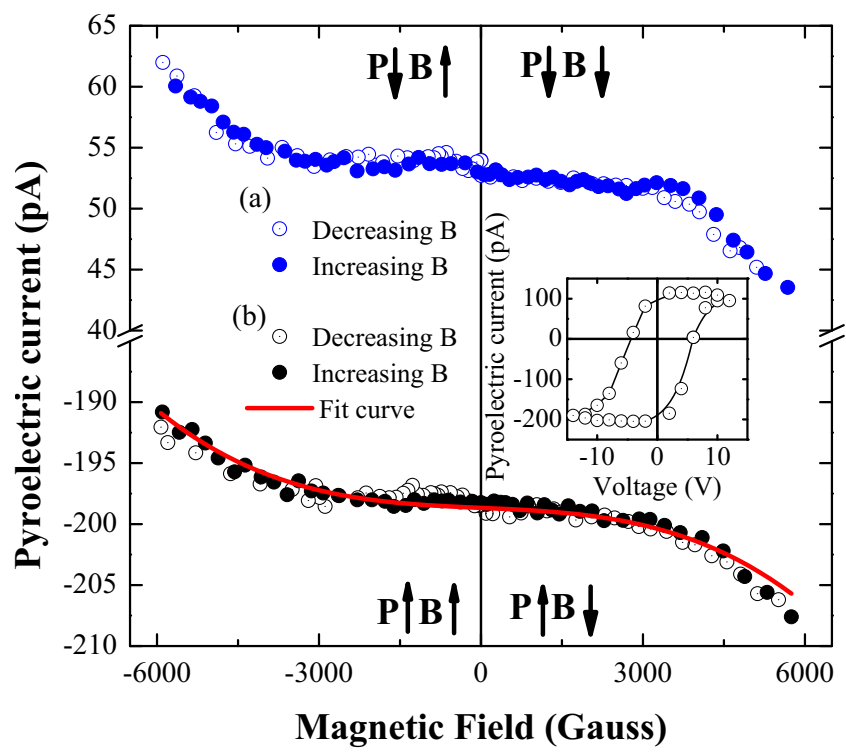

FIG. 2. (Color online) Pyroelectric current (proportional to ferroelectric polarization) as a function of increasing (solid circle) and decreasing (open circle) applied magnetic field perpendicular to the sample plane. The curves labeled (a) and (b) correspond to the two different ferroelectric polarization directions of the sample. Arrows indicate the relative orientation of the polarization $(P)$ and applied magnetic field $(B)$. The solid line is a fit to one set of data (see text). Inset: measurements of the pyroelectric current vs applied voltage showing the ferroelectric polarization hysteresis loop.

rent was measured by a lock-in amplifier locked to the chopper frequency with a time constant of $1 \mathrm{~s}$.

Magnetization measurements were carried out using the polar magneto-optical Kerr effect (PMOKE) which measures the out-of-plane magnetization. To demagnetize the sample, it was mounted on a rapidly rotating drill in a slowly decreasing magnetic field. Out-of-plane PMOKE measurements (Fig. 1) reveal a typical narrow s-shaped hard axis magnetization hysteresis loop, as expected for a $10 \mathrm{~nm}$ Co thin film with an in-plane easy axis. The variously oriented in-plane magnetic domains rotate into the magnetic field direction, forming a cone of half-angle $(\pi / 2-\phi)$ with the sample normal. The slope of the loop gives the angle for the magnetization as a function of applied field, $\sin (\phi)=(1.86$ $\times 10^{-4}$ ) $H$, with $H$ in units of Gauss. Pyroelectric current measurements resulted in the ferroelectric polarization hysteresis loop ${ }^{13}$ shown in the inset of Fig. 2.

After electrical saturation (but prior to magnetic saturation), the pyroelectric response was measured as a function of both increasing and decreasing perpendicular magnetic field sweeps, (Fig. 2) showing an unexpectedly large (over $5 \%$ ) change in the pyroelectric response on application of a 6 $\mathrm{kG}$ magnetic field. The sign of the change in polarization depended on the relative orientation between the polarization and magnetic field. Parallel orientation resulted in a decrease in the absolute value of the polarization with increasing magnetic field, whereas an anti-parallel alignment resulted in an increase. This effect was fully reversible, repeatable and possessed odd symmetry with respect to direction of the out-ofplane magnetic field. We emphasize that this was not an irreversible magnetic poling effect. ${ }^{15}$ Rather, it was a fully reversible and continuous change in the polarization as can be seen in the increasing and decreasing field sweeps.

The measured changes in polarization $\Delta P$ with applied magnetic field $H$ were fitted to a nonlinear ME coupling of the form $\Delta P=\alpha\left(H-H_{s}\right)+\beta\left(H-H_{s}\right)^{3}$. Assuming a saturation

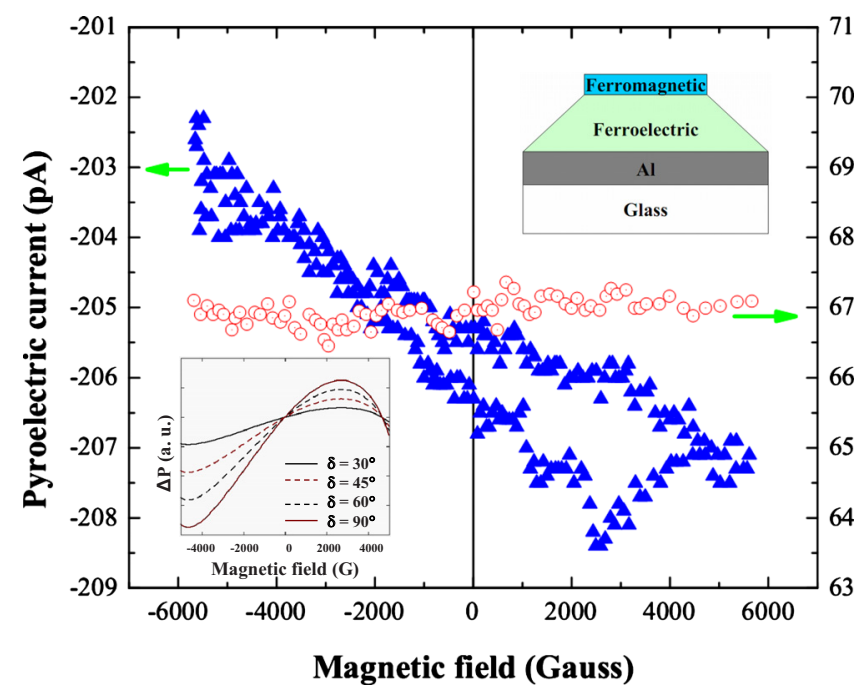

FIG. 3. (Color online) Ferroelectric polarization as a function of applied magnetic field perpendicular to the sample plane, after out-of-plane magnetic saturation (red circles) and after demagnetization (blue triangles). (Upper inset) Schematic diagram of the strain gradient in the ferroelectric layer due to magnetostriction in the upper ferromagnetic electrode. (Lower inset) Calculation [from Eq. (1)] of the changes in polarization with applied field across a single domain wall for various values of $\delta$, the angle between magnetization directions for two adjacent domains.

polarization of $\mathrm{P}(\mathrm{VDF}-\mathrm{TrFE})$ of $0.1 \mathrm{C} / \mathrm{m}^{2}$, we obtain a linear ME coefficient of $\alpha=4.78 \times 10^{-8} \mathrm{C} / \mathrm{m}^{2}$ Oe (equivalent to $\alpha=5.45 \mathrm{~V} / \mathrm{cm} \mathrm{Oe}$ ) and a third-order coefficient of $\beta$ $=7.82 \times 10^{-15} \mathrm{C} / \mathrm{m}^{2} \mathrm{Oe}^{3}$. The linear ME coefficient is comparable to those measured previously in composite materials such as nanopillars ${ }^{3}$ of $\mathrm{BaTiO}_{3}-\mathrm{CoFe}_{2} \mathrm{O}_{4}$ or laminates ${ }^{4}$ of PZT/terfenol-D, an unexpected result given the much smaller magnetostriction of Co.

The data shown in Fig. 2 are for a virgin magnetic sample, with multiple in-plane magnetic domains. After magnetic saturation, either in-plane of or out-of-plane, the polarization shows negligible changes with applied magnetic field, as shown in Fig. 3 for out-of-plane saturation (red circles). The necessity of multiple magnetic domains in the cobalt film is further underscored by the blue triangles symbols in Fig. 3, which show the $P$ versus $H$ curve after demagnetization of the Co film, which restores the effect, albeit at a lower level, approximately $2.5 \%$ instead of $5 \%$ as in Fig. 2. Demagnetization results in smaller domains and hence a higher density of domain walls, as confirmed by magnetic force microscopy imaging on an identically grown Co sample. Demagnetization reduced the average domain size to $200 \mathrm{~nm}$, approximately $1 / 3$ the size of the domains measured after magnetic saturation. Clearly, the presence of multiple domains plays an essential role in this effect. Any discussion of the origin of this large ME effect must account for the dependence on the presence of multiple magnetic domains and the odd symmetry with respect to the directions of the applied magnetic field and the sample polarization. Purely magnetostrictive effects fail on both counts, as well as severely underestimating the magnitude of the change. Since the ferroelectric layer is essentially unclamped in the out-ofplane direction, any changes in the polarization will arise only from changes in the in-plane stress with magnetic field. Using the known values of the elastic and piezoelectric coefficients for $\mathrm{P}(\mathrm{VDF}-\mathrm{TrFE}),{ }^{16}$ and the magnetostriction of Co, purely magnetostrictive strain leads to an estimated rela- 
tive change in the ferroelectric polarization $\Delta P / P$ of order $10^{-4}$, two orders of magnitude smaller than the $5 \%$ measured change. Although the magnetostrictive coefficients in thin films may be three to five times larger than the bulk value, ${ }^{17,18}$ this still fails to account for the large changes in polarization.

An alternative explanation arises from the asymmetric geometry of our sample. Magnetostrictive effects occur only in the Co electrode, inducing a strain gradient in the thickness of the film, $\partial \varepsilon / \partial z$. The flexoelectric effect, ${ }^{19,20}$ defined as the polarization change induced by a strain gradient is given by (neglecting the tensor character of the flexoelectric effect $\left.{ }^{19}\right) \Delta P=-f(\partial \varepsilon / \partial z)$, where $f$ is the flexoelectric coefficient, and $\partial \varepsilon / \partial z$ is the strain gradient in the ferroelectric film (see the upper inset of Fig. 3). Since the strain gradient $\partial \varepsilon / \partial z$ would be largest for a uniformly magnetized cobalt film, and smallest for a demagnetized film, this is also inconsistent with of the results shown in Fig. 3.

However, the presence of multiple in-plane magnetic domains means numerous in plane strain gradients exist in the magnetic film and consequently in the ferroelectric film. These could contribute to the changes in polarization via the flexoelectric effect. The strain gradient across the domain wall between two in-plane magnetic domains oriented at an angle $\delta$ with respect to each other is given by $\partial \varepsilon / \partial x$ $=\left[\left(3 \lambda_{s} / 2 W\right)\left(\cos ^{2} \delta-1\right)\right]$, where $W$ is the domain wall width. The changes in the magnitude of the polarization resulting from these in-plane strain gradients are governed by the components of the flexoelectric coefficient tensor $f_{i j k l}{ }^{21,22}$ For a single crystallite of the ferroelectric polymer, the $x_{3}$ axis is parallel to the polarization tilted at $30^{\circ}$ away from the film normal. It can easily be shown that the only relevant strain gradient is $\partial \varepsilon_{3} / \partial x_{3}$. As the out-of-plane magnetic field is increased, the magnetization within each domain rotates toward the magnetic field direction, making an angle with the sample plane given by $\phi(H)$ (obtained from the magnetic hysteresis loop). This changes the angle between the two adjacent domains and hence changes the strain gradient across the domain wall which is now given by

$$
\frac{\partial \varepsilon_{3}}{\partial x_{3}}=\frac{3 \lambda_{s}}{4 W} \cos ^{2}\left[120^{\circ}-\phi(H)\right]\left[\cos ^{2} \delta-1\right] .
$$

The configuration of domains, including their density and the in-plane angle $\delta$ between adjacent domains results in a net strain gradient, and together with the magnitude of the flexoelectric coefficient (unknown for P[VDF-TrFE]) will determine the magnitude of the change in the polarization. Since the configuration of domains is highly sensitive to roughness, structure, and magnetization history the change in the magnitude of the effect after demagnetization is not surprising. A plot of Eq. (1) as a function of applied field and for various values of $\delta$, the angle between two adjacent domains, is shown in the lower inset of Fig. 3. In a perfectly demagnetized isotropic sample, all values of $\delta$ are equally probable; in reality there will be a preference for certain angles, depending on the microstructure of the Co layer. A more sophisticated calculation would take into account a distribution function for the angle $\delta$, the density of domains and the net strain gradient that exists across the macroscopic area of the sample.
In conclusion, we have demonstrated a large converse ME effect in a heterostructure composed of a ferromagnetic cobalt film and a ferroelectric polymer film. Application of a magnetic field to the heterostructure results in changes in the electric polarization of up to $5 \%$. The result is highly dependent on the presence of multiple magnetic domains in the cobalt film. We propose a model in which the interaction arises from strain gradients at the magnetic domain walls coupling to flexoelectric response in the polymer layer. These results provide a qualitative look into the phenomenon; quantitative study will require independent measurement probing key features of the underlying mechanisms including the flexoelectric response of the ferroelectric polymer, the effect of the magnetic state in the ferroelectric hysteresis loops, the domain-wall strain of the ferromagnetic film, and the evolution of the magnetic domains with magnetic field. The sensitivity of this effect to the exact domain configuration of the ferromagnet may provide a path for tuning both the magnitude and symmetry of the effect.

This research is supported by the National Science Foundation through the Materials Research Science and Engineering Center program under Grant No. DMR-0820521.

${ }^{1}$ V. E. Wood and A. E. Austin, Int. J. Magn. 5, 303 (1974).

${ }^{2}$ T. Kimura, T. Goto, H. Shintani, K. Ishizaka, T. Arima, and Y. Tokura, Nature (London) 426, 55 (2003).

${ }^{3}$ H. Zheng, J. Wang, S. E. Lofland, Z. Ma, L. Mohaddes-Ardabili, T. Zhao, L. Salamanca-Riba, S. R. Shinde, S. B. Ogale, F. Bai, D. Viehland, Y. Jia, D. G. Schlom, M. Wuttig, A. Roytburd, and R. Ramesh, Science 303, 661 (2004).

${ }^{4}$ Ce-Wen Nan, N. Cai, Z. Shi, J. Zhai, G. Liu, and Y. Lin, Phys. Rev. B 71 014102 (2005).

${ }^{5}$ K. Mori and M. Wuttig, Appl. Phys. Lett. 81, 100 (2002).

${ }^{6}$ J. Zhai, S. Dong, Z. Xing, J. Li, and D. Viehland, Appl. Phys. Lett. 89, 083507 (2006).

${ }^{7}$ Z. Fang, S. G. Lu, F. Li, S. Datta, Q. M. Zhang, and M. El Tahchi, Appl. Phys. Lett. 95, 112903 (2009).

${ }^{8}$ S. G. Lu, Z. Fang, E. Furman, Y. Wang, Q. M. Zhang, Y. Mudryk, K. A. Gschneidner, V. K. Pecharsky, and C. W. Nan, Appl. Phys. Lett. 96, 102902 (2010).

${ }^{9}$ A. V. Bune, V. M. Fridkin, S. Ducharme, L. M. Blinov, S. P. Palto, A. V. Sorokin, S. G. Yudin, and A. Zlatkin, Nature (London) 391, 874 (1998).

${ }^{10}$ S. Palto, L. Blinov, A. Bune, E. Dubovik, V. Fridkin, N. Petukhova, K. Verkhovskaya, and S. Yudin, Ferroelectrics Lett. 19, 65 (1995).

${ }^{11}$ J. Choi, C. N. Borca, P. A. Dowben, A. Bune, M. Poulsen, S. Pebley, S. Adenwalla, S. Ducharme, L. Robertson, V. M. Fridkin, S. P. Palto, N. N. Petukhova, and S. G. Yudin, Phys. Rev. B 61, 5760 (2000).

${ }^{12}$ B. J. Rodriguez, S. Jesse, S. V. Kalinin, J. Kim, and S. Ducharme, Appl. Phys. Lett. 90, 122904 (2007).

${ }^{13}$ A. G. Chynoweth, J. Appl. Phys. 27, 78 (1956).

${ }^{14}$ A. V. Bune, C. Zhu, S. Ducharme, L. M. Blinov, V. M. Fridkin, S. P. Palto N. G. Petukhova, and S. G. Yudin, J. Appl. Phys. 85, 7869 (1999).

${ }^{15}$ V. R. Palkar, K. Ganesh Kumara, and S. K. Malik, Appl. Phys. Lett. 84, 2856 (2004).

${ }^{16} \mathrm{P}(\mathrm{VDF}-\mathrm{TrFE}) \quad \mathrm{c}_{11}=3.5 \times 10^{9} \mathrm{~N} / \mathrm{m}^{2}=\mathrm{c}_{22} \quad$ and $\quad \mathrm{c}_{12}=\mathrm{c}_{21}=\mathrm{c}_{13}=\mathrm{c}_{23}=1.2$ $\times 10^{9} \mathrm{~N} / \mathrm{m}^{2}$, and $\mathrm{d}_{3 \mathrm{j}}=\mathrm{d}_{32}=4.3 \mathrm{pC} / \mathrm{N}$.

${ }^{17}$ Y. K. Kim and T. J. Silva, Appl. Phys. Lett. 68, 2885 (1996).

${ }^{18}$ O. Song, C. A. Ballentine, and R. C. O' Handley, Appl. Phys. Lett. 64, 2593 (1994)

${ }^{19}$ W. Ma, Phys. Scr. T129, 180 (2007).

${ }^{20}$ A. K. Tagantsev, Phys. Rev. B 34, 5883 (1986)

${ }^{21} \mathrm{We}$ assume that over the width of a ferromagnetic domain wall, the ferroelectric consists of a single crystal, with a unique direction of polarization. Domain walls in Co, approximately $30 \mathrm{~nm}$ are comparable to the ferroelectric crystallites, $30 \mathrm{~nm}$ to $50 \mathrm{~nm}$ (Ref. 12). The presence of multiple ferroelectric crystallites within a magnetic domain wall will reduce the effect.

${ }^{22}$ J. F. Nye, Physical Properties of Crystals (Oxford University Press, New York, 1985). 